\title{
An Engineering Accreditation Management System
}

\author{
Steven K. Dew \\ Faculty of Engineering \\ University of Alberta \\ steven.dew@ualberta.ca
}

\author{
Mathieu Lavoie, Ashley Snelgrove \\ Castle Rock Research Corporation \\ mlavoie@castlerockresearch.com \\ asnelgrove@castlerockresearch.com
}

\begin{abstract}
We have developed a web-based system to assist in the management of the engineering accreditation process. It provides a centralized tool for curriculum analysis, course and instructor database management, program information collection and tracking, report preparation, and graduate attribute planning and monitoring.
\end{abstract}

\section{Introduction}

The accreditation process [1] under the Canadian Engineering Accreditation Board (CEAB) is extremely thorough and time-consuming, involving a multi-year timeframe and the exhaustive collection, organization and analysis of information. We crudely estimate that the last review of the University of Alberta's (UofA) nine programs required the collection of more than a tonne of documents and consumed approximately $16,000 \mathrm{~h}$ in preparation time involving a personnel cost of well over $\$ 1 \mathrm{M}$. Further, the people most tied up in these preparations are the Faculty's senior leadership who are thereby unable to advance the strategic and operational objectives of the organization. Hence, there is a tremendous potential benefit of a system that would help simplify the task of managing engineering accreditation.

Meeting this challenge is compounded by recent $\mathrm{CEAB}$ changes to link instruction of engineering content with professional licensure through the socalled Qualified Accreditation Units (AU's). This means that relatively static program curricula must be linked with much more dynamic annual teaching assignments in order to demonstrate compliance. However, the 2008 introduction of the twelve graduate attributes and the requirement to implement a system to measure and continuously improve graduate outcomes will have the most significant impact on how Canadian faculties of engineering respond to accreditation requirements. This is very similar to the ABET process [2] employed in the United States, which has posed a significant adjustment to American schools over the last decade. It also means that accreditation is no longer an episodic task undertaken once every six years, but is now an ongoing continuous activity.

In response to these challenges, the UofA has partnered with educational resource company Castle Rock Research Corporation [3] to develop a webbased software tool to greatly simplify the task of collecting, analyzing and presenting the information necessary to demonstrate compliance with the CEAB requirements. We call this the Accreditation Management System (AMS).

\section{Requirements of the AMS}

Clearly, one of the requirements of the AMS will be to analyze curricula to ensure that CEAB minima are being met in all categories. Traditionally, this has been done using spreadsheets. With elective streams and options, at the UofA there are 30 separate program specifications that must be analyzed across four departments. This is extremely difficult to maintain consistently using spreadsheets, especially with the recent Qualified AU's where the teaching assignment for a service course in one department will impact the accreditation in another. Hence, one main requirement is that a single centralized database be used to hold all course, instructor and program information so that consistency of data and approach can be maintained across the faculty. For reasons of privacy protection, that database must sit behind a firewall within the institution's own network. Nonetheless, that data must be easy to enter, modify, review, and track.

Further, we would like the application to be available to a wide range of administrators and instructors so that tasks and responsibilities can be distributed to the appropriate level. This necessitates a web-based user interface so that there is no requirement for specialized software to be deployed and maintained at the desktop. With a multiuser approach also comes a need for access control (id and 
password) and varying user privileges based on their respective roles. This, along with an audit tracker, will help ensure the integrity of the data. The distributed, multiuser approach also mandates a robust system with an intuitive user interface along with online help to ensure infrequent users can be productive and not frustrated.

Of course, the AMS system must meet the needs of the $\mathrm{CEAB}$ reporting. Along with curriculum analysis, that means generating course information sheets, instructor CV's, licensure summaries, course content summaries, curriculum committee lists, resource summaries, etc. In all, there are 30 different tables and forms required in a CEAB report that should be generated by the AMS system in the correct format.

One important area for which CEAB has not yet specified how to report relates to graduate attributes. Taking an approach similar to that typically used for ABET reporting, for each of the twelve attributes, the AMS system should provide a framework for generating curriculum maps, specifying performance indicators, scheduling and inputting measurement data, recording analysis results, and generating archival reports to document an overall continuous improvement process. This topic will be discussed further in section 4.6 .

\section{Implementation of the AMS}

Levering the capabilities and expertise of Castle Rock, the AMS Solution implemented using the C\# language within the Microsoft .NET framework. Database services are provided under SQL, web services under IIS and Apache Tomcat and content management using an in-house tool developed by Castle Rock. The full set of software needed is listed below:

- Windows Server 2008 R2

- $\quad$ Microsoft SQL Server 2005

- Apache Tomcat

- $\quad$ IIS 7

- ASP.NET 3.5

The hardware required to run the AMS application consists of a Windows Server 2008 R2 based system with a dual core processor and 4GB of memory. Storage requirements vary with data needs, but would typically be met by 1 TB for a large, multi-program institution.

\section{Capabilities}

The current capabilities of the AMS system are demonstrated in the sections below.

\subsection{User Roles}

Within the AMS system, there are a variety of roles a user can be assigned. Faculty Admin users have the highest privileges and can access any data or create/delete any kind of user. Typical Faculty Admin users would be Associate Deans or others coordinating at the Faculty level. Department Admin users have authority only within their own unit in which they can create or modify programs or courses, or create Instructor or other Department Admin users. They would also make teaching assignments and review accreditation checklists. Typically, these would be Chairs, Associate Chairs, and senior departmental administrators. Most users of the AMS would be Instructors who can access only their assigned courses and modify their own personal data and CV's. This hierarchical structure ensures appropriate access to sensitive information and minimizes the chance of inadvertent corruption of data. Of course, all users are authenticated by a password and transactions are logged for review if problems arise.

\subsection{Course Information}

The biggest single source of data for the AMS relates to courses. At the UofA, we have almost 500 courses relevant to our undergraduate programs. Each requires all of the information needed to generate a CEAB course information sheet, including the breakdown of AU's into the various categories (Math, Natural Sciences, Complementary Studies, Engineering Science, Engineering Design, Other), plus the list of instructors, plus various content from the course (syllabus, notes, exams, labs, graded student work, web pages, etc.) that can be uploaded to form a repository for future reference as well as to satisfy program visitors before and during their visit.

Within the AMS, the main page for entering course information is as shown in Figure 1. Tabs along the left hand side allow the user to jump to different sections to review or enter course information. An index page allows navigation between courses and can be sorted by faculty and department or searched based on course number or title. A teaching assignment page allows the selection of instructors within a department with regard to their PEng status and the needs of the course in the context of Qualified AU's.

Course data can also be imported from a commaseparated value (CSV) file of text data. This allows much of the course information to be input directly from reports generated by existing academic information systems. Course data is associated with a given Academic Year, and most of it automatically rolls forward when a new Academic Year is created. 


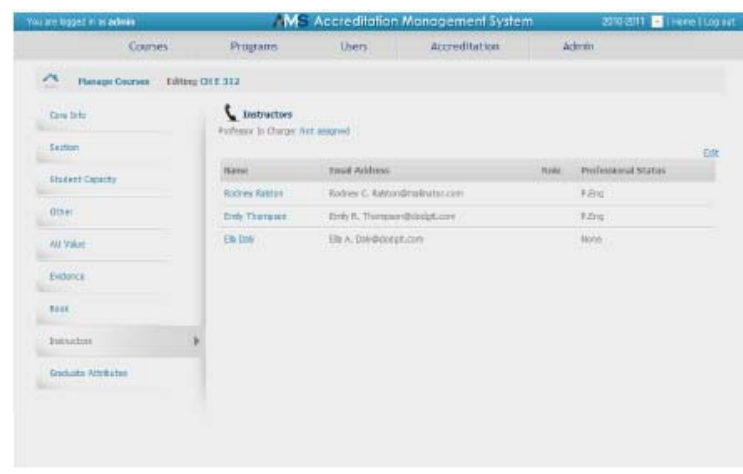

Figure 1. Main page for course data entry.

Gathering course information and ensuring it is complete is a major, tedious task when preparing for a CEAB visit. To assist in this, the AMS system provides a checklist functionality to review what has been input and uploaded onto the system. An example checklist is shown in Figure 2.

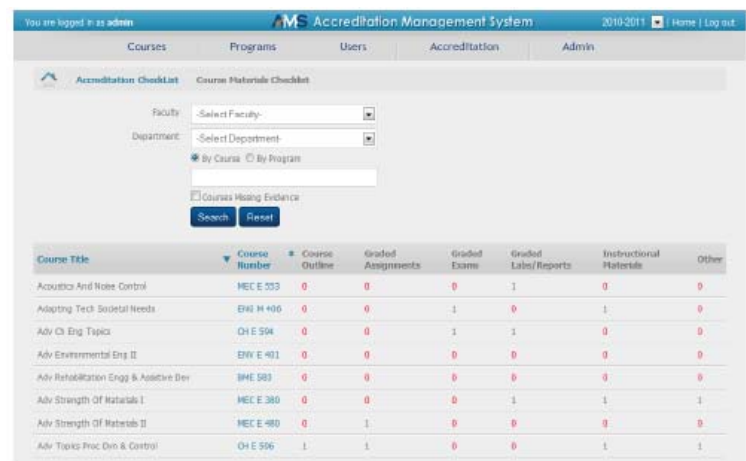

Figure 2. Example checklist page for tracking course information.

\subsection{Instructor Information}

Within the Faculty of Engineering at the UofA, there are almost 250 individuals involved in instruction. Their credentials and status must be recorded, particularly to be able to track Qualified AU's and generate CV's and other CEAB tabular reports. This information is typically input by the Instructor who can also upload a CV in PDF format. Figure 3 shows the main Instructor information page. As with the course information page, there are tabs along the left side that allow quick navigation to enter specific information. As well, there is a CV status page that Admin users can use to review which Instructors have completed the entry process.

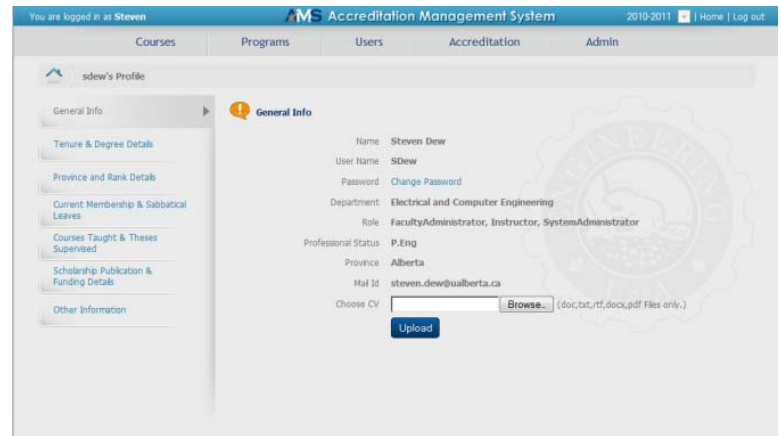

Figure 3. Main page for instructor information entry.

\subsection{Program Information}

A key component of CEAB accreditation is a quantitative 'minimum path' analysis of the program curriculum against a number of content requirements. This requires specification of common core, program compulsory, option compulsory and elective courses. This specification is typically done by a Department or Faculty Admin user via the Program Builder page shown in Figure 4. Tabs near the top allow navigation through the different categories (e.g. program compulsory) and tables at the top track the AU's accumulated in each of the content types. Electives are specified in groups with selection rules such as "choose two out of the following..."

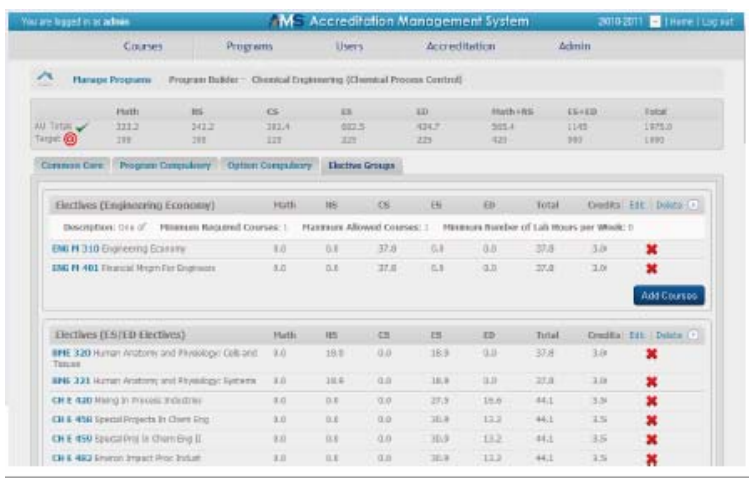

Figure 4. Program Builder page for specifying the courses required for a given program or option.

Reports on the program AU content can be selected from a list allowing assessment at different levels of detail. Qualified AU's are displayed at this level. These consider the licensure status when counting Engineering Science (ES) and Engineering Design (ED) content. Under recent CEAB rules, ED only counts towards Qualified ED AU's if it is being taught by an instructor with a PEng. ES only counts towards the Qualified ES+ED total if it is taught by a PEng or a recent hire (within 5 years) actively pursuing registration [4]. Hence, program totals must link into instructor assignments and instructor 
qualifications and appointment date for determining these totals. Of course, the minimum path principle applies here, so if multiple sections exist, only the least qualified instructor counts towards this calculation. This highlights the need to carefully consider program AU totals when making teaching assignments.

As well, program specific information such as staff changes, contents summaries, enrolment data, transcripts or expenditures can be input and reviewed within these program reports.

A quick summary of curriculum content for all programs can be found on the main program management page. Figure 5 shows an example with multiple programs and options. Values are colour coded as green (compliant with CEAB requirements), yellow (over the minimum but by less than $10 \%$ ) or red (under CEAB minimum).

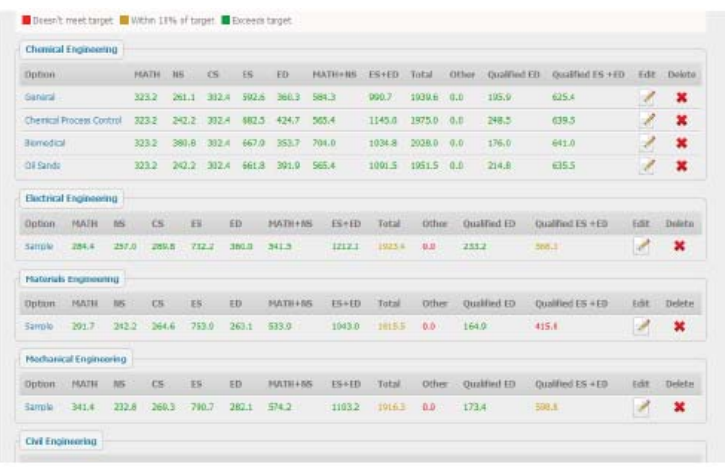

Figure 5. Main program management page with a summary of curriculum totals.

\subsection{CEAB Reports}

The purpose of the AMS is to facilitate accreditation. Hence, it should generate reports in the format expected by CEAB. In addition to the instructor CV's and course information sheets, the AMS system currently generates the CEAB tables listed below. This is basically all of the mandatory tables in the questionnaire. They are generated on a program-byprogram basis in PDF format.

Table 1. List of CEAB tables generated by the AMS.

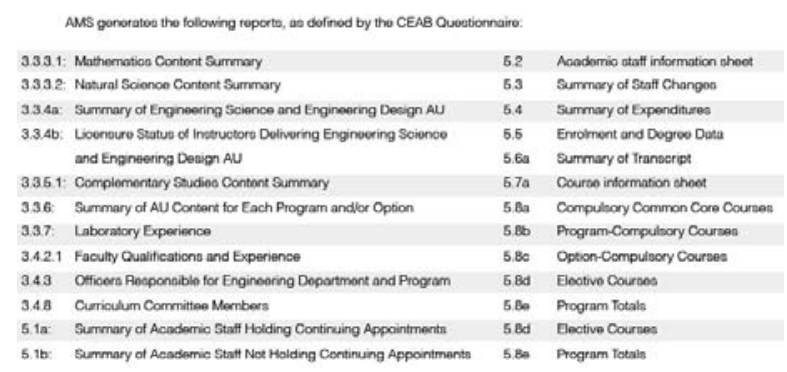

\subsection{Graduate Attributes}

Since 2008, CEAB has introduced the additional requirement that programs demonstrate that their students possess certain attributes at the time of graduation. These attributes are listed in Table 2. Furthermore, these attributes are to be measured as part of a continuous improvement process, necessitating a systematic approach. Such a process can be greatly facilitated by a system like the AMS.

Table 2. CEAB graduate attributes

\begin{tabular}{|c|l|c|l|}
\hline 1 & Knowledge Base & 7 & Communication skills \\
\hline 2 & Problem Analysis & 8 & Professionalism \\
\hline 3 & Investigation & 9 & Society \& Environment \\
\hline 4 & Design & 10 & Ethics and Equity \\
\hline 5 & Engineering Tools & 11 & Economics \\
\hline 6 & Team Work & 12 & Lifelong Learning \\
\hline
\end{tabular}

The CEAB has not at this time defined a standard approach for implementing a graduate attribute process. Groups such as the EGAD project [5] sponsored by the National Council of Deans of Engineering and Applied Sciences have developed some resources to facilitate this task, and the ABET system provides a model that can be emulated.

Borrowing from the ABET model, the UofA has developed an approach that employs a number of performance indicators (PI's) for each of the 12 graduate attributes. These are not intended to be exhaustive measures of the attribute, but provide an indication of whether the requisite characteristics associated with the attribute have been developed. These PI's are to be objectively measurable and usually associated with a rubric defining levels of achievement. As an example, a subset of the PI's and rubrics developed by the UofA for its Communication Skills attribute is presented in Figure 6.

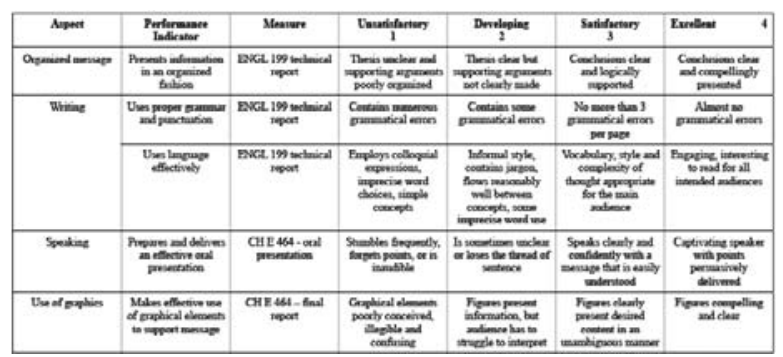

Figure 6. Partial set of performance indicators for the Communication Skills graduate attribute.

The AMS system provides a framework for specifying the PI's and associated courses, rubrics and targets. These, of course, will depend on the program. Based on course information, the AMS can generate a map showing where and the degree to which the 
attributes are developed across the curriculum. This assists in choosing courses in which measurements can be taken. (See Figure 7) Further, it can provide a process for scheduling when and who will perform the assessments. Measured data can be analyzed and recommendations recorded in a systematic manner. These outcomes can be captured in a report that documents the continuous improvement process. Figure 8 shows a model of such a report with multiyear data for various PI's related to the Team Work attribute.

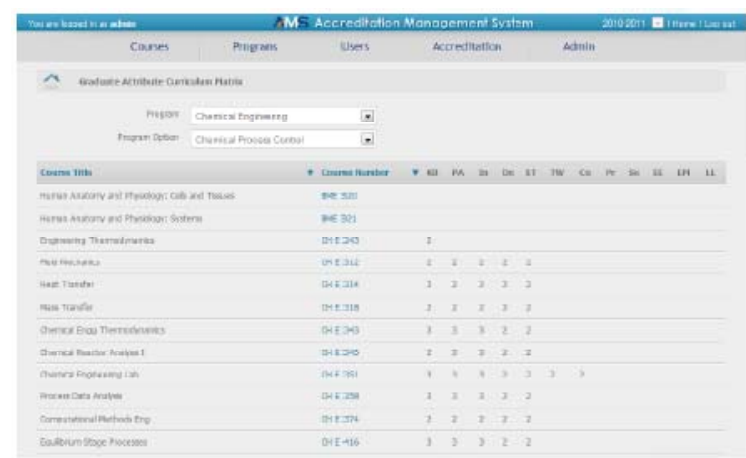

Figure 7. Attribute map showing the degree each of the 12 attributes are developed in each of the courses in a program.

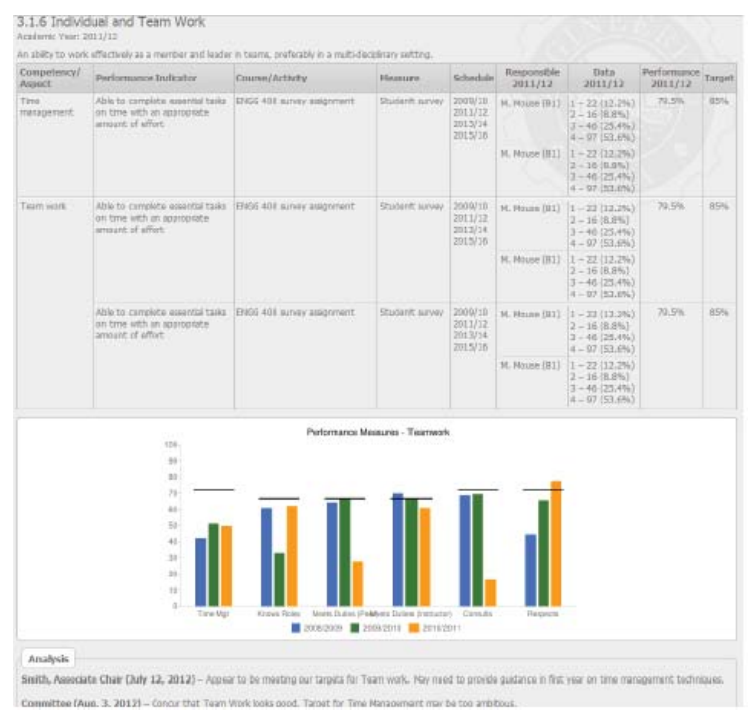

Figure 8. Model report for the Team Work attribute showing the schedule for measuring the individual PI's along with the current date, multiyear trends against the target performance, along with the outcomes of the analysis.

\section{Conclusions}

An accreditation management system has been developed which facilitates the collection, organization, analysis and presentation of the data required to demonstrate compliance with $\mathrm{CEAB}$ requirements. Preliminary indications are that the AMS is very effective, particularly given the growing complexity of accreditation with the introduction of Qualified AU's and graduate attributes. These latter requirements move accreditation from something worried about every 6 years to something that continuously permeates the delivery of our programs.

\section{Acknowledgments}

The authors would like to acknowledge the work of their UofA colleagues developing graduate attribute performance indicators and measures, and the efforts of the Castle Rock team developing the AMS application. We also gratefully acknowledge the financial support of the Alberta Department of Advanced Education and Technology for this project.

\section{References}

[1] 2010 Canadian Engineering Accreditation Board Accreditation Criteria and Procedures, Engineers Canada http://www.engineerscanada.ca/e/files/Accreditation_ Criteria_Procedures_2010.pdf

[2] 2011-12 Accreditation Policy and Procedure Manual, ABET

http://www.abet.org/Linked\%20DocumentsUPDATE/Program\%20Docs/abet-appm-20112012.pdf

[3] Castle Rock Research Corporation http://www.castlerockresearch.com

[4] Statement of interpretation on licensure expectations and requirements, Appendix 3, 2010 Canadian Engineering Accreditation Board Accreditation Criteria and Procedures, p. 55.

[5] Engineering Graduate Attribute Development (EGAD) Project http://engineering.queensu.ca/egad 